\title{
Anesthetic challenges in cancer patients: considerations for palliative management of tracheoesophageal fistula: report of two cases
}

\section{L.J. Pinzón Lozano1, B.J. Escobar Suarez²}

1. Anesthesiology residency program, National University of Colombia. Bogotá, Colombia

2. Department of Anesthesiology, National University of Colombia, National Cancer Institute. Bogotá, Colombia

Background:The palliative management of patients with tracheoesophageal fistula (TEF) is a challenge to the anesthesiologist who despite the abnormality of the anatomy and function of the airway, apnea and endoscopic manipulation must keep the patient with spontaneous ventilation, normoxemia and normocapnia.

Case1: 43 year old patient with metastatic breast cancer who presents TEF by false path during a tracheostomy. Under sedation with dexmedetomidine, the tracheotomy cannula was removed. FTE was observed, via bronchoscopy, high-frequency ventilation (Jet) was initiated, and introduced self-expandable covered stent. In the intensive care unit, stent was placed, patient presented hypoxemia. didn't respond to treatment and suffered a cardiac arrest.

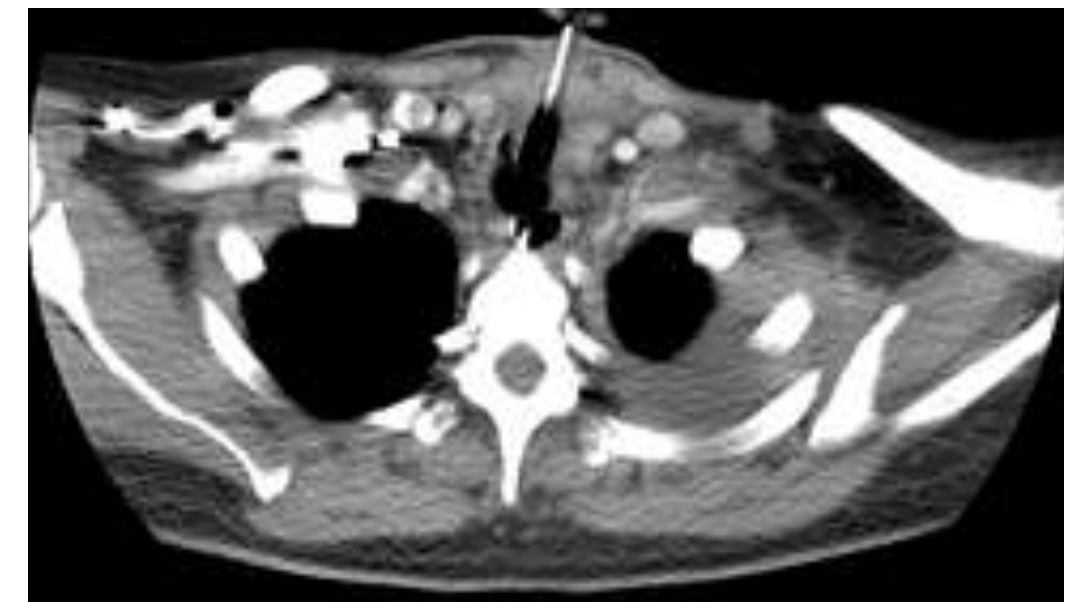

Picture 1. TEF of $3.6 \mathrm{~mm}$

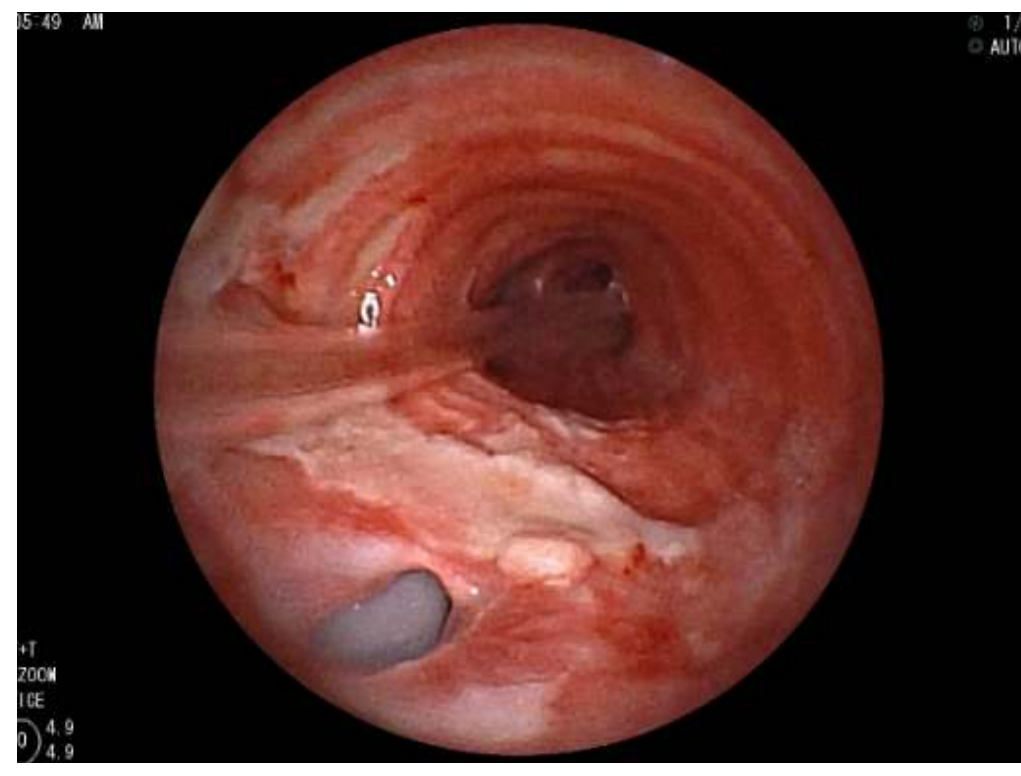

Picture 2. TEF of $20^{\star} 10 \mathrm{~mm}$ - jet.

\section{Case 2:}

57 year old patient with stage IIA esophageal squamous cell cancer who required chemotherapy, radiotherapy and an esophageal stent, was admitted for aspiration pneumonia and suspected TEF.

Fibrobronchoscopy evidenced TEF at the left bronchus. The esophageal stent was removed and patient was scheduled for a dual endoscopic procedure. Patient was sedated with dexmedetomidine and both (bronchial and esophageal) stents were placed. Utilizing the Karnofsky Performance scale the patient scored 90/100 with a previous rating of 60/100.

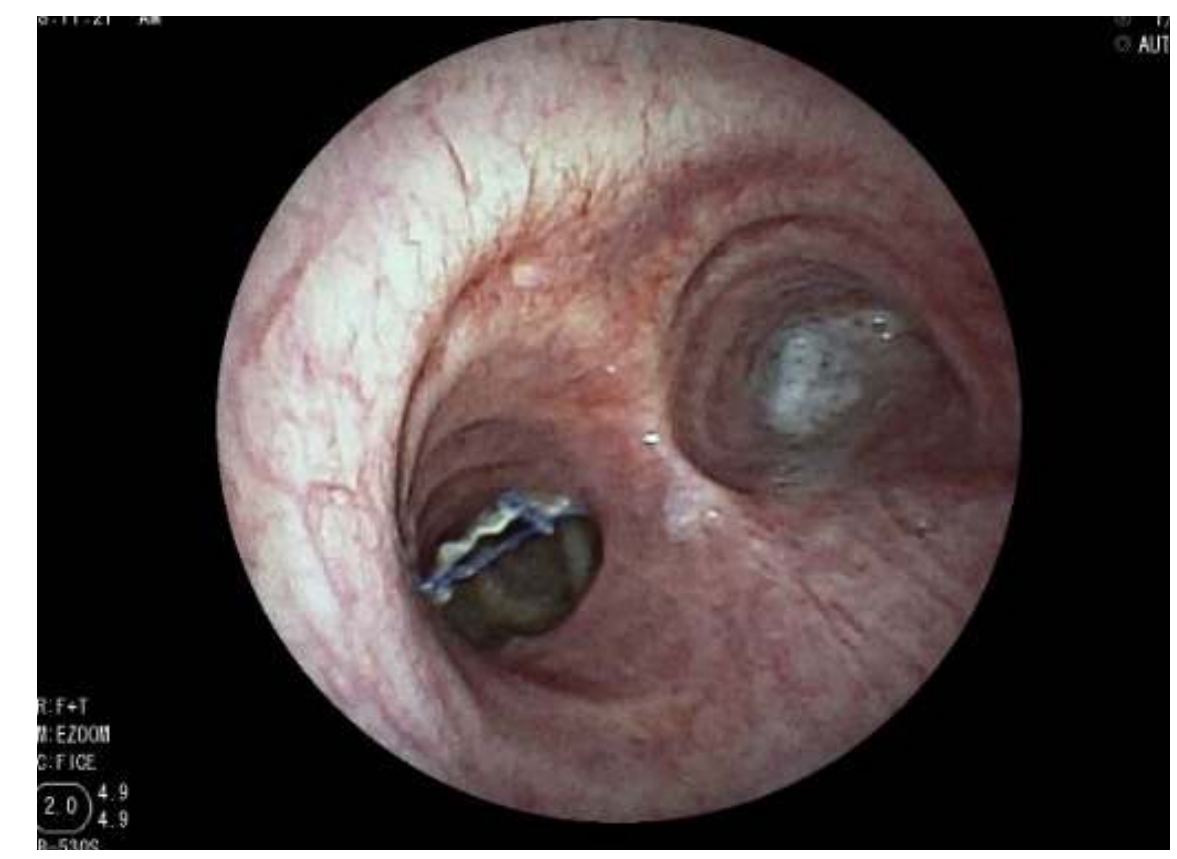

3. TEF of $15 \mathrm{~mm}$. Esophageal stent at left bronchus

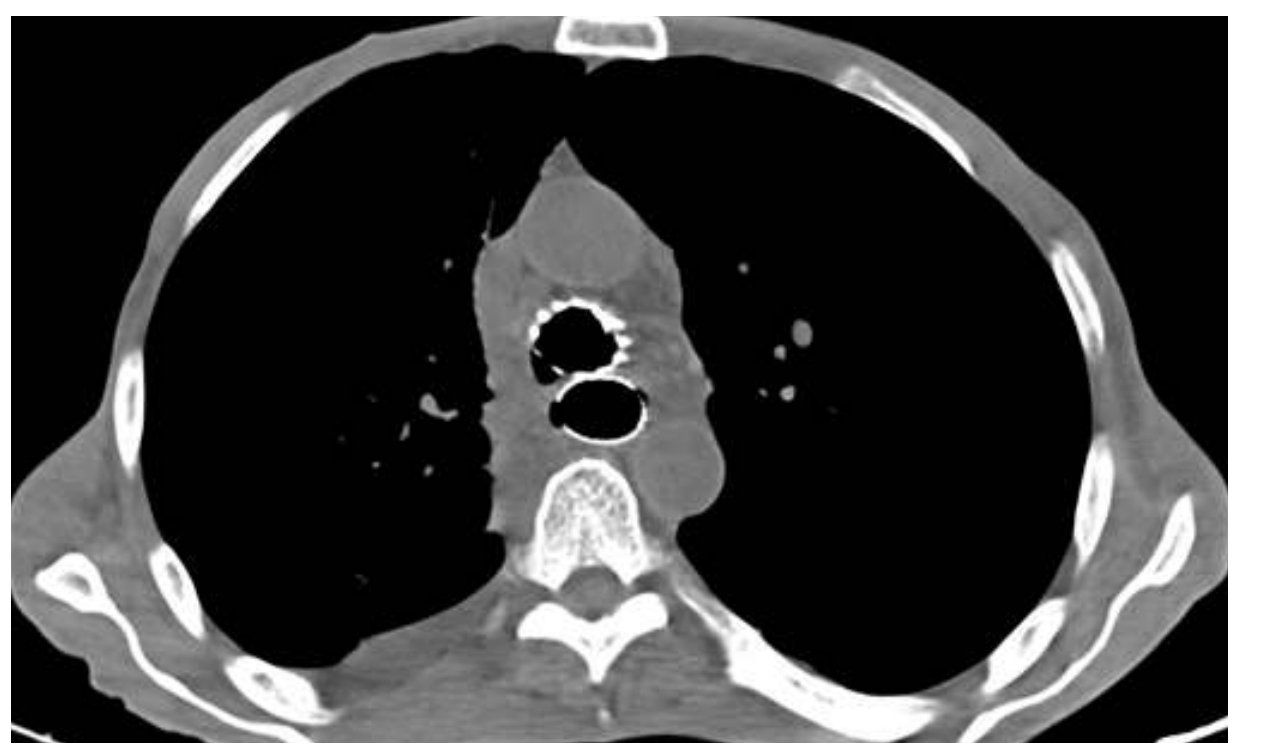

Picture 4 bronchial and esophageal stent

Results and Discussion: In the intraoperative, considering that these patients with decreased lung compliance and function, increasing the difficulty for ventilation after anesthetic induction presented by hypoventilation, hypoxemia and hypercapnia. Muscle relaxation and positive pressure ventilation should be avoided, and patient's spontaneous breathing decrease the risk of bronchoaspiration and prevent the escape of volume by TEF. Total intravenous anaesthesia using propofol or dexmedetomidine is recommended. In cases of difficult oxygenation and ventilation, high frequency ventilation can be used as an adjuvant. The oxygen administration should be titrated to the minimum tolerable by maintaining an adequate flow of fresh air with $\mathrm{FiO} 232-40 \%$, after period of apnea should be administrated $100 \%$.

Conclusions:The anaesthesiologist should avoid muscle relaxation, positive pressure ventilation, and inhaled agents.

References: 1.Semin Cardiothorac Vasc Anesth.2017;21:357-359. 